\title{
USING FIBER-OPTIC SENSORS AND 3D PHOTOGRAMMETRIC RECONSTRUCTION FOR CRACK PATTERN MONITORING OF MASONRY STRUCTURES AT THE AURELIAN WALLS IN ROME, ITALY
}

\author{
IRENE BELLAGAMBA ${ }^{1}$, MICHELE CAPONERO $^{2}$ \& MARIALUISA MONGELLI ${ }^{3}$ \\ ${ }^{1}$ Student Grant, Department of Energetic Technologies, ENEA, Rome, Italy \\ ${ }^{2}$ Researcher, Department of Fusion, ENEA, Rome, Italy \\ ${ }^{3}$ Researcher, Department of Energetic Technologies, ENEA, Rome, Italy
}

\begin{abstract}
This paper shows an application of the photogrammetric 3D reconstruction by SfM (Structure from Motion) technique and Fiber Bragg Grating (FBG) sensors to evaluate the long-term crack propagation and the damage evolution on the Aurelian Walls in Rome. Aurelian Walls were built between 270 and 275 A.C. by the Emperor Aureliano to defend Rome, the capital of the Empire, from barbaric attacks. Originally, they extended for about $19 \mathrm{~km}$, nowadays remains are $12.5 \mathrm{~km}$ long and among the longest and best-preserved ancient wall murals in the world. The two adopted techniques offer complementary advantages. By the SfM reconstruction it is possible to acquire the geometry of the studied masonry structure and to detect most relevant cracks where FBG sensors can be installed for permanent monitoring. Moreover, SfM allows to acquire the crack pattern over extended surfaces and to compare its evolution with scheduled repeated measurements. FBG sensors allow continuous monitoring at selected critical locations and offer reference data for correlation of scheduled photogrammetry measurements. 3D Photogrammetric reconstruction by SfM took advantage of hardware and software capabilities of the HPC (High Performance Computing) resources available in ENEA, which are provided by the CRESCO (Research Computational Centre on Complex Systems) infrastructure. FBG sensors were installed in thermal compensation configuration and with both high stability for long-term static measurements and dynamic response capabilities. Experimental data so far acquired are presented with evidence of the preliminary results of the measurement campaign, which is planned to be continued in the long term.
\end{abstract}

Keywords: structural monitoring, fiber-optic sensors, photogrammetry, SfM technique.

\section{INTRODUCTION}

This work began with the "COBRA" [1] project, but it is currently being developed within "ADAMO" [2] and "ECODIGIT" [3] projects financed by the Lazio Region, that promote the development and dissemination of methods, technologies and tools for cultural heritage protection. This work shows the use of 3D photogrammetric reconstruction by SfM as a very fast and low cost technique to produce numerical models based on 2D images, to support structural investigations in terms of crack patterns definition [4], [5], to define stress/strain distributions and to evaluate dynamic behaviour of the structures to be monitored [6].

This work shows the case study of the monumental structure of the Aurelian Walls, focused on one of the guarding towers (namely the "L3 tower") where crack patterns had already been subjected to continuous monitoring by FBG sensors for about one year in 2010 .

A 3D photogrammetric reconstruction of the L3 tower was acquired so that the "reference status" was fixed to investigate the evolution of the damage over the time by both scheduled $3 \mathrm{D}$ photogrammetric reconstructions to be regularly repeated in the long-term continuous monitoring by FBG sensors currently being installed on the most critical pointed out cracks. The 3D photogrammetric reconstruction, performed by PhotoScan pro and Meshlab software, was used to build a FE model and to execute the modal analysis in order to obtain 
the first modal shapes of the structure. The FE model was obtained by Patran and the preliminary FE modal analysis by Nastran, while the definition of the cracking framework was performed by AutoCAD. Software packages are available via ENEAGRID [7] through the Virtual LAB ITACHA.

The work aims to propose this approach describing advantages and weaknesses of the photogrammetric technique compared to other NDTs and, in particular, it suggests the employment of FBG sensors as an integrative survey for both continuous monitoring at selected locations and the calibration of the parameters used in the adopted numerical models.

\section{THE 3D PHOTOGRAMMETRIC RECONSTRUCTION BY SFM}

The 3D Photogrammetric reconstruction by SfM technique [8] allows for the obtainment a three-dimensional model of an object or of a structure starting from the acquisition of two-dimensional digital images. This technique is mainly based on the principles of optics, photography and descriptive geometry, that allow to resolve some analytical relations to determinate a univocal correspondence between the points located in the so called "object space" (images space) and the points of the real 3D space.

The SfM has many advantages: it's a fast, low-cost, not invasive and not destructive technique, which executes the moving scan of any structure in a "contactless" way. The SfM technique [9], [10] can also be used to create, in a semi-automatic way, the finite element model of a complex structure, in order to execute the finite element analysis, taking into account its real geometric properties.

\subsection{Photogrammetric technique by $\mathrm{SfM}$ - general principles}

From the acquisition of two-dimensional digital images, the photogrammetric technique by SfM allows us to obtain a 3D model of an existing structure, under the form of "points cloud" or "polygonal mesh", the cameras position and the cameras orientation within the space, in order to detect its correct measures in terms of shape, size and space position. The SfM technique is an evolution of the traditional photogrammetric method, since the scene reconstruction, the camera position and orientation, are automatically resolved by the SfM software.

Each reconstruction step uses specific algorithms that are able to recognise the "principal features" or "remarkable points" of an object and then a database is created. On the base of the detected features, an "image matching" process is performed: it consists in grouping all the images that have some points in common. The "bundle adjustment" process, based on the so-called "Collinearity equations", allows to detect the "features positions" and the cameras orientation (internal/external).

The result of this step is a "sparse point cloud" model, consisting of the features detected previously, i.e. the points characterised by three spatial coordinates and a chromatic information obtained from images.

Subsequently, a "dense image matching algorithm" extracts a "dense point cloud" model, thanks to a depth comparison between images. A vector and a triad of chromatic values (RGB: Red Green Blue) are associated to each point belonging to the dense cloud.

Some photo-modelling software can also reconstruct a polygonal model, made of triangular elements, faces and vertices (meshes), and then a "texture mapping" process allows creating a final "polygonal texturized" model of the structure, thanks to the information obtained from the camera orientations.

One of the principal obstacles for the use of the SfM technique is due to the large demands of hardware and software resources for image processing, data analysis and data storage. The 
possibility to access the computational resources offered by the ENEAGRID infrastructure, through the so called FARO2 (Fast Access to Remote Objects) graphical interface, allows to use "computer graphics" tools, exploiting the computational resources offered by the HPC CRESCO infrastructure [11] and to archive images and photogrammetric reconstruction results in the AFS and GPFS ENEA storage areas. The photogrammetric reconstructions described in this paper are all made-up by the use of the computer vision software named PhotoScan pro, available via ENEAGRID through the Virtual LAB ITACHA installed on CRESCO (Fig. 1).

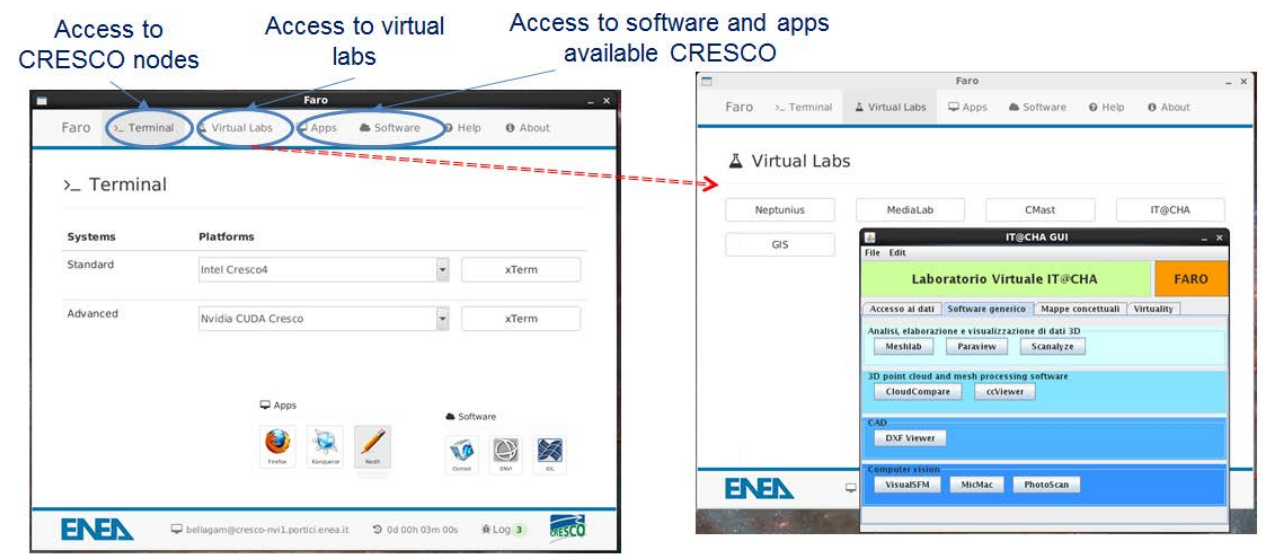

Figure 1: Remote access to PhotoScan pro software.

\subsection{The SfM reconstruction software performed by ENEAGRID: "PhotoScan pro"}

Thanks to the use of the computational resources offered by CRESCO, PhotoScan pro software can manage a large number of images, with the possibility to create high-quality polygonal models, characterized by a multi-view reconstruction.

The image post-processing procedure is based on "Computer Vision" algorithms, it is easy and semi-automatic, and it can be resumed as shown in Fig. 2.

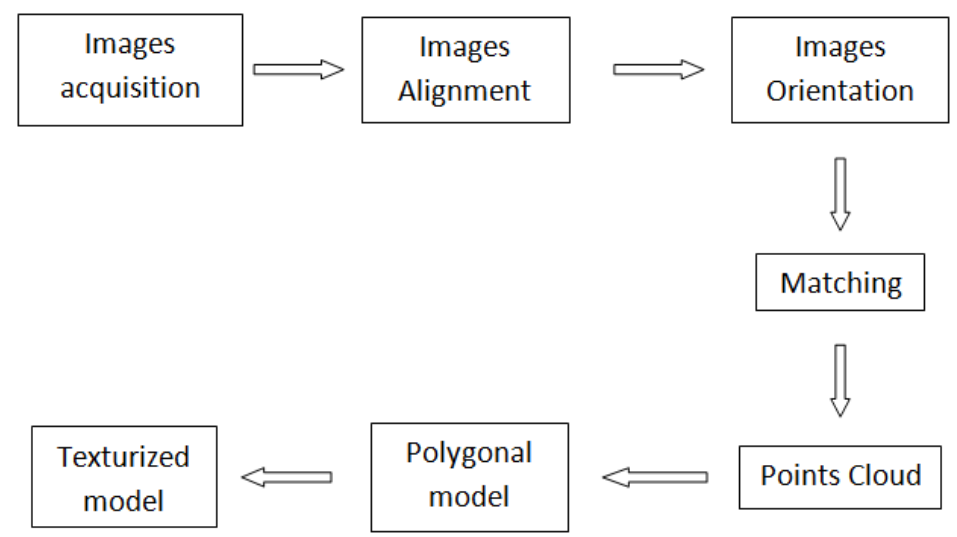

Figure 2: 3D reconstruction process - PhotoScan pro workflow. 
The first step of the elaboration process performed by PhotoScan pro is the alignment of the loaded images: by means the SIFT algorithm and the multi-stereo matching process, the position and the orientation of the cameras are detected and a "sparse cloud" model is created. Subsequently, a "dense image matching" algorithm allows to extract the "dense cloud" model, thanks to a depth comparison between all the images.

PhotoScan pro also allows reconstructing the polygonal model, made of faces and vertices, which can be obtained from the points belonging to the dense point cloud. Then, a texture process is performed, in order to obtain the final "Texturized model". There are two different methods to create the texture, the "mosaic method", based on the projection of the image with the best resolution on each portion of the model and the "average method", which takes into account the average value of the single pixel characterizing all the images.

With the aim to detect the real dimensions of the detected structure for the execution of the FE analysis, a scaled polygonal model is needed. For this purpose, the PhotoScan pro software provides a fast and automatic way to scale the $3 \mathrm{D}$ model. starting from the acquisition and the assignment of a single reference measure of the real structure.

\section{CASE STUDY: THE "L3 TOWER"}

The "L3 tower" is the second tower at the west side of Porta San Sebastiano, one of the doors of the Aurelian Walls. It is reachable by the Museum of the Walls, through a walkway (Fig. 3). The tower is accessed from the east wall with a small opening to the outside; the north wall has a large window, while the south wall is characterized by a single small slit. The walk continues through the west wall, exactly mirrored to the east wall and it is characterized by a door and a small alley. On the top of the tower, at about six and a half meters high, a portion of huge vault is partially collapsed.

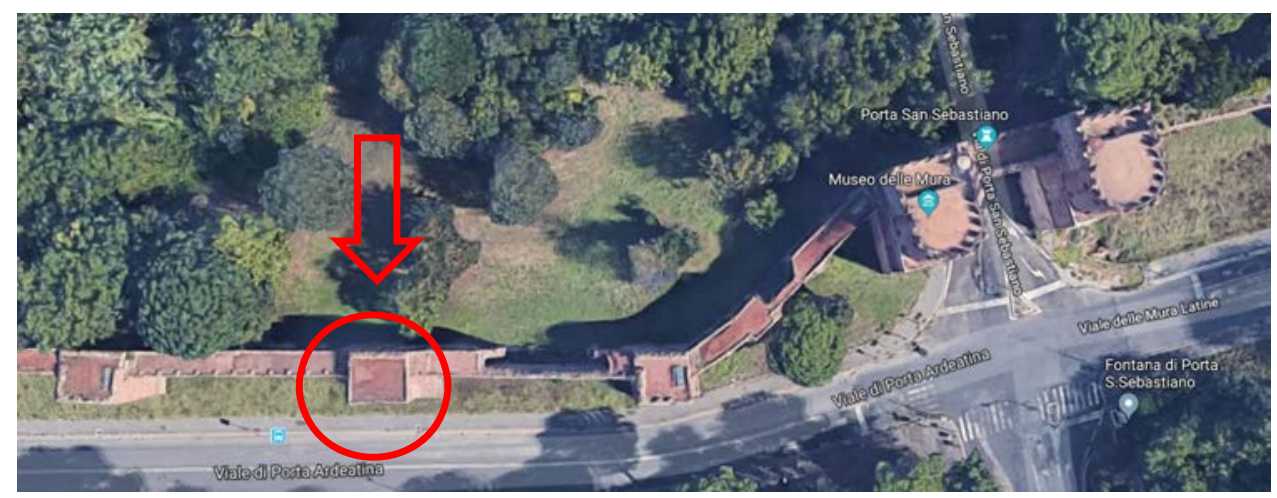

Figure 3: Location of the L3 tower.

\subsection{Structural monitoring by SfM technique}

The photogrammetric relief consists of $2932 \mathrm{D}$ digital images, each with a resolution of 3872 x 2592 pixels, taken by Nikon D60 with a mean shooting distance of approximately $3 \mathrm{~m}$ and a focal length of $18 \mathrm{~mm}$.

The first goal was to obtain a three-dimensional global model of the Tower L3 by SfM technique using Photoscan Pro, scaling the model by the real geometric dimensions and building a $3 \mathrm{D}$ model as faithfully as possible to reality, to be used for preliminary FE analysis. 
The scaled polygonal model of the entire L3 tower, performed by PhotoScan pro, has been imported in the CAD software in a standard "dxf" file extension. In AutoCAD we changed the file format, from "dxf" to "stl", in order to obtain an export file that is suitable for executing a preliminary FE modal analysis.

Fig. 4 shows the complete 3D CAD model of the tower represented from four different sides: North, South, East and West.

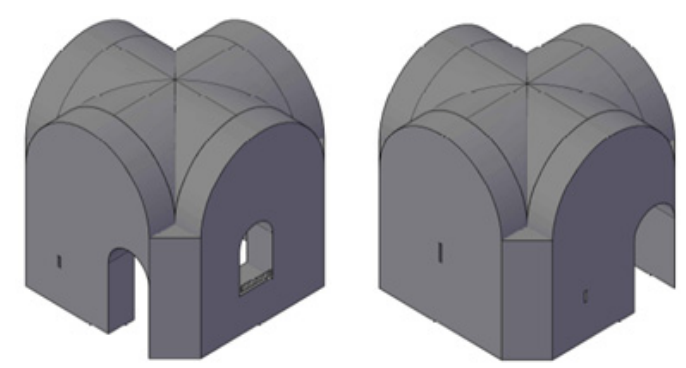

(a)

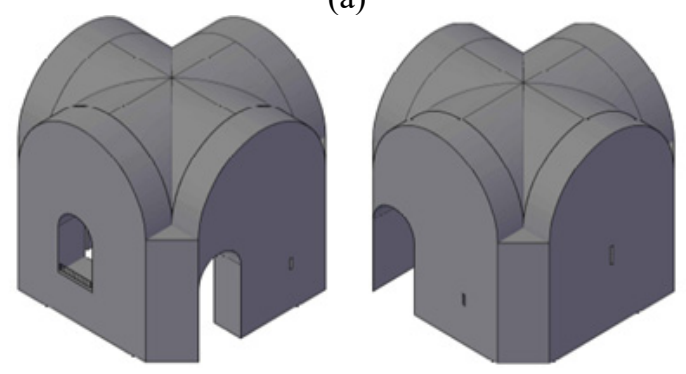

(b)

Figure 4: AutoCAD 3D model of the L3 tower. (a) East-West side; and (b) North-South side.

The modal analysis was performed by NASTRAN code in order to detect the fundamental modal shapes of the L3 tower allowing to identify the critical areas to be monitored by Fiber Bragg Grating (FBG) sensors [12] during future measurement campaigns. The model was joined to the base with a fixed support. In Table 1, we reported the mechanical characteristics of the material assigned to the FE model.

Table 1: Material properties of the structure.

\begin{tabular}{|l|c|c|}
\hline \multicolumn{3}{|c|}{ Material properties } \\
\hline Property & Value & Units \\
\hline Young Modulus & 1800 & $\mathrm{MPa}$ \\
\hline Density & 1800 & $\mathrm{Kg} / \mathrm{m}^{3}$ \\
\hline Poisons coefficient & 0.15 & - \\
\hline
\end{tabular}

The results of the finite element modal analysis of the tower, in terms of modal shapes, are shown in Fig. 5. 

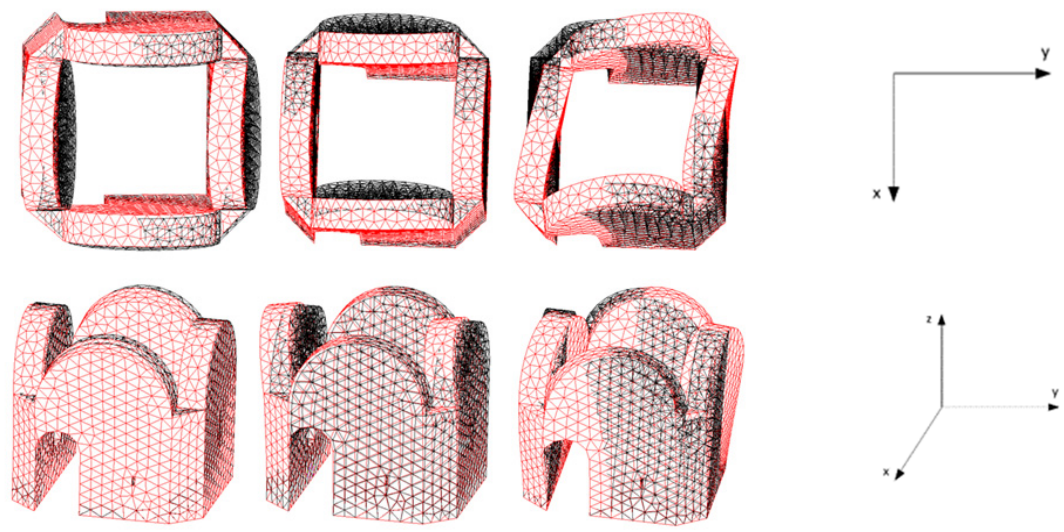

Figure 5: Modal shapes of the L3 tower: (left) 1st mode - longitudinal x; (centre) 2nd modal - transversal y; (right) 3rd mode - torsional z.

Table 2: Frequencies of the first three modal shapes of the structure.

\begin{tabular}{|c|c|}
\hline \multicolumn{2}{|c|}{ Modal analysis } \\
\hline \multicolumn{2}{|c|}{ Frequencies [Hz] } \\
\hline 1st mode & 12 \\
\hline 2nd mode & 12.5 \\
\hline 3rd mode & 21.6 \\
\hline
\end{tabular}

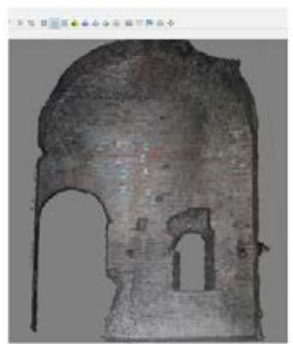

EST side

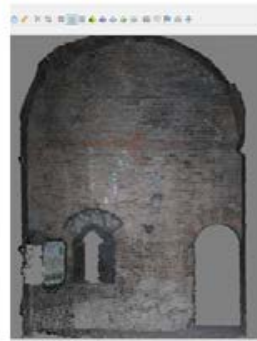

WEST side

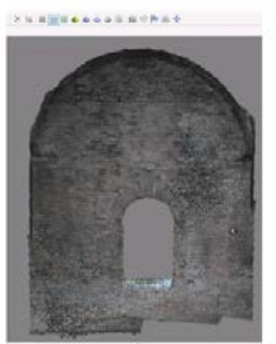

NORTH side

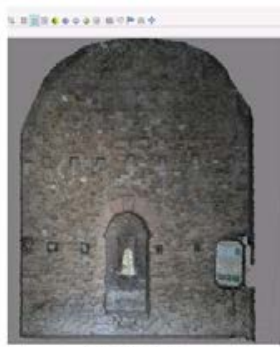

SOUTH side

Figure 6: Dense cloud points of the walls.

The frequencies of the first three modal shapes (longitudinal, transversal and torsional) are resumed in Table 2.

At the same time, for each wall a dense cloud was created (Fig. 6) with the aim to reconstruct and define the complete cracking framework of the EAST and WEST wall.

Two "orthomosaic images", representing the "dense cloud" models of the two walls, were produced from PhotoScan and then imported in AutoCAD, in order to reconstruct the entire crack pattern (Fig. 7) of the two walls. 

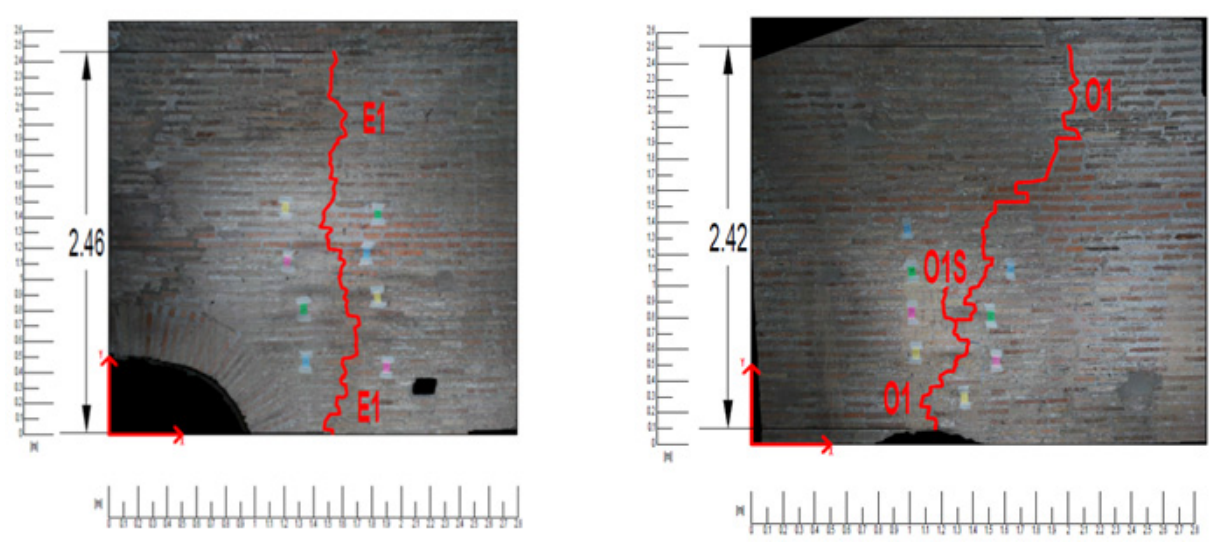

Figure 7: Crack patterns detection.

The resolution of the 3D model depends on many factors including the shooting distance, the focal length, the images resolution and the size of the camera sensor. These factors are related to the so-called Ground Sample Distance (GSD), which represents the real physical size of the object that a single pixel represents in the image and it gives an information about the reconstruction's resolution.

The resolution of each acquired image is 5184 x 3456 pixels, whose average shooting distance is estimated to be $2.5 \mathrm{~m}$. Considering the size of the sensor, equal to $15.8 \mathrm{~mm}$, and the focal distance of the lens, equal to $18 \mathrm{~mm}$, we obtained the following values:

\begin{tabular}{|c|c|c|}
\hline GSD $(\mathrm{mm} / \mathrm{px})$ & $\mathrm{D}_{\mathrm{H}}(\mathrm{mm})$ & $\mathrm{D}_{\mathrm{V}}(\mathrm{mm})$ \\
\hline 0.34 & 1.756 & 1.170 \\
\hline
\end{tabular}

where $D_{H}$ and $D_{V}$ respectively represent the width and height of the image footprint on the photographed object. The GSD is equal to $0.34 \mathrm{~mm} / \mathrm{px}$ that represents the resolution of the model.

The map of the cracks was completely described (Table 3), in terms of extension and damage category, according to the macro-seismic European EMS98, also used by the 2nd GNDT level cards, considering five levels of damage (low, moderate, high, very high, collapse). The damage category was assigned on the basis of cracks' extension, that is evaluated to be more than $2 \mathrm{~m}$ (cracks code E1 and O1). As shown in Fig. 7, the cracks start from the openings of the L 3 tower. These cracks may have occurred as a result of a weakening of the load-bearing arch system of the openings, due to the detachment of some blocks constituting the arc structure.

Table 3: Crack features and dimensions.

\begin{tabular}{|l|c|c|c|c|c|c|}
\hline \multirow{2}{*}{$\begin{array}{l}\text { Crack } \\
\text { code }\end{array}$} & \multicolumn{2}{|c|}{ Start coordinates } & \multicolumn{2}{c|}{ End coordinates } & Crack extension & $\begin{array}{c}\text { Damage } \\
\text { category }\end{array}$ \\
\cline { 2 - 5 } E1 & $\mathrm{X}_{\mathrm{i}}[\mathrm{m}]$ & $\mathrm{Y}_{\mathrm{i}}[\mathrm{m}]$ & $\mathrm{X}_{\mathrm{f}}[\mathrm{m}]$ & $\mathrm{Y}_{\mathrm{f}}[\mathrm{m}]$ & 2.46 & 2 \\
\hline O1 & 0.29 & 0.01 & 0.3 & 2.47 & 2.46 & 2 \\
\hline O1S & 0.37 & 0.17 & 1.21 & 2.58 & 2.42 & 1 \\
\hline
\end{tabular}


This is only a preliminary survey of crack pattern that wants to show how the SfM technique can automatically detect the length and exact position of cracks. Other following monitoring surveys are needed to completely understand the pathologies of the tower.

\subsection{Structural monitoring by FBG sensors}

The main crack patterns identified by SfM analysis will be permanently monitored by FBG sensors whose positioning planning and installation is currently in progress. FBG sensors will be installed as crackmeters. Crackmeters are made with a slim harmonic-steel lamina, to be anchored at both ends across the crack: two FBG sensors are present on the lamina, one acting as a strain gauge and thus providing measurement of crack opening via lamina elongation, the other one acting as a temperature sensor and thus allowing data taking in temperature compensation mode. Here, we briefly recall that FBG sensors are fibre-optic sensors suitable for both temperature and strain sensing, that many sensors can be connected in-series thus allowing minimally invasive cabling, that they do not require power supply at the measuring point, that they allow very stable long-term measurement in both quasi-static and dynamic regime. A comprehensive review of FBG sensors principles and applications is given in Kersey [12]. The two cracks shown in Fig. 7 have already been subjected to continuous monitoring by FBG sensors for about one year in the 2010; just one crackmeter per crack was installed, at about its median height.

Fig. 8 shows a sample of acquired data; data are given with temperature compensation, so that they effectively show the pure geometric opening of the cracks.

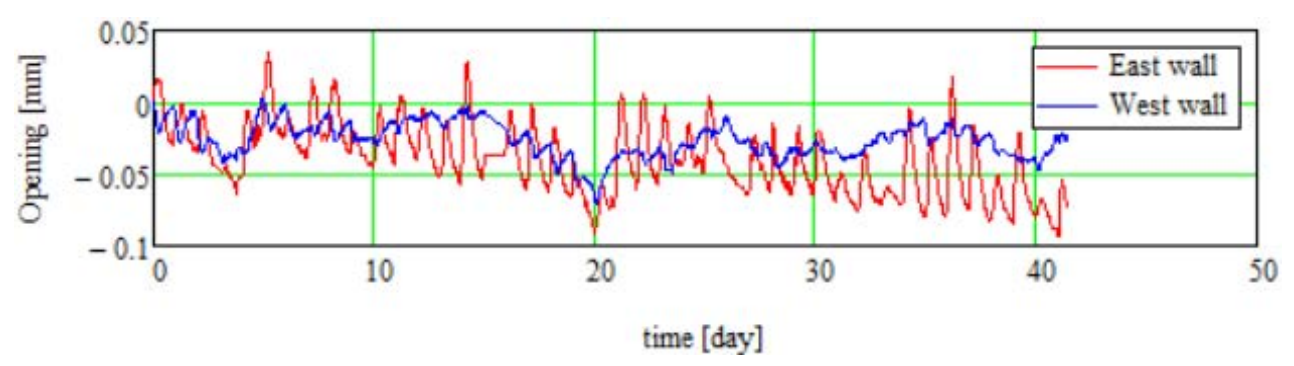

Figure 8: Time history of crack opening - continuous monitoring interval of 42 days.

Time history of crack openings clearly shows correlation with day/night cycle, pointing out quite a different amplitude for the two opposite walls. To be noted the out-of-phase occurrence of the maxima opening for the two walls, which evidently is a consequence of the cardinal orientation.

\section{CONCLUSIONS}

The aim of this paper is to propose a very fast methodology to support the protection of cultural heritage by monitoring the evolution of modal properties and crack patterns. The methodology was successfully applied on the case study of the L3 tower at the Aurelian Walls. It is based on a 3D photogrammetric restitution by means the SfM technique, starting from the acquisition of 2D digital images.

The reconstructed 3D geometrical model allows to produce a FE model via CAD, which can be used to study the dynamic structural behaviour of the structure. The 3D model also allows to point out critical crack patterns, which can be permanently monitored by 
crackmeters whose data can be useful for both tuning FE parameters and correlation of long term scheduled 3D photogrammetric surveys.

The advantages in using the SfM technique for cracks monitoring, if compared with the simple visual inspection and other NDTs tests, is that it is a non-contact, automatic and fast way to execute the survey. Moreover, this technique permits to obtain a direct 3D model of the real structure that can be subsequently modified and updated on the bases of cracks evolution without the need to draw again the model "by hand".

In the case study, hardware and software resources were available thanks to the ENEA ICT computing infrastructure. Moreover, FBG sensors were adopted as crackmeters due to their long-term stability capabilities which will reliably allow the application of the proposed methodology in the long time.

\section{REFERENCES}

[1] CO.B.RA., Project, Rome, http://cobra.enea.it/english.

[2] ADAMO, Project, Rome, http://progettoadamo.enea.it/.

[3] ECODIGIT, Semantic Technology Laboratory (STLAB). http://stlab.istc.cnr.it/stlab/project/ecodigit/.

[4] Mongelli, M., De Canio, G., Roselli, I., Malena, M. Nacuzi, A. \& de Felice, G., 3D photogrammetric reconstruction by drone scanning for FE analysis and crack pattern mapping of the bridge of the Towers, Spoleto. Key Engineering Materials, 747, pp. 423-430, 2017.

[5] Mongelli, M. et al., Structure-from-Motion (SfM) technique in the Catacombs of Priscilla in Rome. Improvements in the conservation, the safety and the fruition. Kermes, 107, pp. 111-116, 2017.

[6] Arias, P., Herraez, J., Lorenzo, H. \& Ordonez. C., Control of structural problems in cultural heritage monuments using close-range photogrammetry and computer methods. Computers and Structures, 83, pp. 1754-1766, 2005.

[7] Abate D. et al., ENEAGRID Infrastructure. High Performance Computing on CRESCO Infrastructure: Research Activities and Results 2010-2011, Italian National Agency for New Technologies, Energy and Sustainable Economic Development 9, 2011.

[8] Westoby, M.J., Brasington, J., Glasser N.F., Hambrey M.J. \& Reynoldsc J.M., Structure from motion photogrammetry: A low-cost, effective tool for geoscience applications. Geomorphology, 179, pp. 300-314, 2012.

[9] McCarthy, J., Multi-image photogrammetry as a practical tool for cultural heritage survey and community engagement. Journal of Archeological Science, 43, pp. 175185, 2014.

[10] Yilmaz, H.M., Yakar, M., Gulec, S.A. \& Dulgerler, O.N., Importance of digital closerange photogrammetry in documentation of cultural heritage. Journal of Cultural Heritage, 8, pp. 428-433, 2007.

[11] Ponti, G. et al., The role of medium size facilities in the HPC ecosystem: the case of the new cresco4 cluster integrated in the ENEAGRID infrastructure. Proceedings of the 2014 International Conference on High Performance Computing and Simulation, pp. 1030-1033, 2014.

[12] Kersey, A., A review of recent developments in fiber-optic sensor technology. Optical Fiber Technology, 2, pp. 291-317, 1996. 